\title{
Tunable Carbon-Dot-Based Dual-Emission Fluorescent Nanohybrids for Ratiometric Optical Thermometry in Living Cells
}

 \\ ${ }^{\dagger}$ China-Australia Joint Research Centre for Functional Molecular Materials, School of Chemical \& Material Engineering, Jiangnan \\ University, Wuxi 214122, People's Republic of China \\ ${ }^{\ddagger}$ Research School of Chemistry, Australian National University, Canberra, ACT 2601, Australia
}

Supporting Information

\begin{abstract}
The use of carbon-dot-based dual-emission fluorescent nanohybrids (DEFNs) as versatile nanothermometry devices for spatially resolved temperature measurements in living cells is demonstrated. The carbon dots (CDs) are prepared in the organic phase and display tunable photoluminescence (PL) across a wide visible range by adjusting the excitation wavelengths and extend of N-doping. DEFNs are formed in a straightforward fashion from CDs (emitting blue PL) and gold nanoclusters (AuNCs, emitting red PL). The DEFNs display ideal single-excitation, dual-emission with two wellresolved, intensity-comparable fluorescence peaks, and function in optical thermometry with high reliability and accuracy by exploiting the temperature sensitivity of their

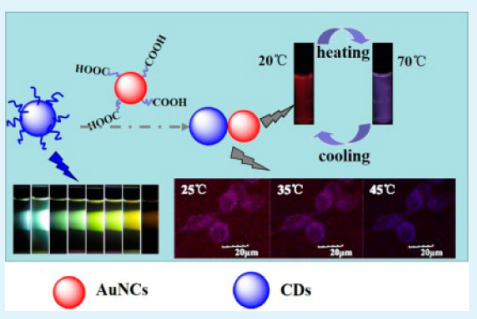
fluorescence intensity ratio (blue/red). Furthermore, the DEFNs have been introduced into cells, exhibiting good biocompatibility, and have facilitated physiological temperature measurements in the range of $25-45{ }^{\circ} \mathrm{C}$; the DEFNs can therefore function as "non-contact" tools for the accurate measurement of temperature and its gradient inside a living cell.
\end{abstract}

KEYWORDS: carbon dots, tunable fluorescence, dual-emission nanohybrid, nanothermometry, living cells

\section{INTRODUCTION}

Temperature is one of the most frequently measured parameters that governs biological reaction within living cells. $^{1-3}$ The accurate measurement of temperature and its gradient inside a living cell can promote advancements in cell biology and biomedicine. ${ }^{4-6}$ To this end, fluorescence-based temperature sensors have shown great potential since they operate as "non-contact" tools and offer the dual functions of cellular imaging and temperature sensing at the molecular level. ${ }^{7-9}$ Many promising fluorescent nanomaterials, such as quantum dots, carbon nanomaterials, rare earth-doped nanoparticles and copper nanoclusters, have been exploited. ${ }^{10-13}$ These optical temperature sensors rely on changes in luminescence intensity as the reporting mechanism (for example, an increase in nonradiative decay rate with temperature leading to decreasing luminescence). ${ }^{11,13}$ However, intensity-based temperature sensors are often susceptible to errors due to changes in probe concentration, the efficiency of excitation or detection, or a lack of specificity for temperature (for example, luminescence may be quenched by oxygen, sensor aggregation, or changes in $\mathrm{pH}$, in addition to the aforementioned reduction due to temperature increase). ${ }^{14,15}$ Changes in the sensor local environment are difficult to control in complicated systems such as cells or microdevices, and can thereby cause inaccurate temperature measurement. Compared with them, dual-emitting sensors show resolvable luminescence emissions from two different excited states. ${ }^{16,17}$ Thus, dualemitting temperature sensors have attracted much attention since they operate as "non-contact" tools and accurately measure temperature. ${ }^{18}$ Several dual-emitting nanomaterials, such as $\mathrm{CdSe} / \mathrm{ZnS}$ nanocrystals, rare earth-doped nanoparticles, and polymer dots have shown potential for nanothermometry in biological systems, especially living cells, ${ }^{19-21}$ but high toxicity or instability has limited their further application.

Carbon dots, as an important class of photoluminescence (PL) nanomaterials, have been the subject of extensive research over the past decade because of inherent advantages such as low cost, reduced toxicity, biocompatibility, and excellent photoresponse. $^{22-24}$ Carbon dots (CDs) have been applied widely in the fields of bioimaging, printing inks, photocatalysis, and sensors. ${ }^{25-29}$ Thus, far, CDs have been prepared by both top-down and bottom-up strategies in the aqueous phase. ${ }^{30,31}$ However, such water-based syntheses may suffer from a very broad size distribution (so an additional size selection step is required) and low product yield. Although dual-emitting nanomaterials based on CDs or metal nanoclusters (NCs) have been shown to function as nanosensors for ascorbic acid, $\mathrm{Cu}^{2+}, \mathrm{Hg}^{2+}$, and highly reactive oxygen species, ${ }^{16,32-34}$ there have been no reports thus far of $\mathrm{CD} / \mathrm{NCs}$-based dual-emitting probes for ratiometric temperature detection in living cells. The emissions of most CDs are centered in the blue and green regions, ${ }^{35-38}$ which may be unfavorable for biological applications because of the harm that short-wavelength radiation may cause to living cells or biosystems. The synthesis of $\mathrm{CDs}$ with broad-range tunable fluorescence therefore remains a major challenge.

Received: November 23, 2015

Accepted: February 24, 2016

Published: February 24, 2016 

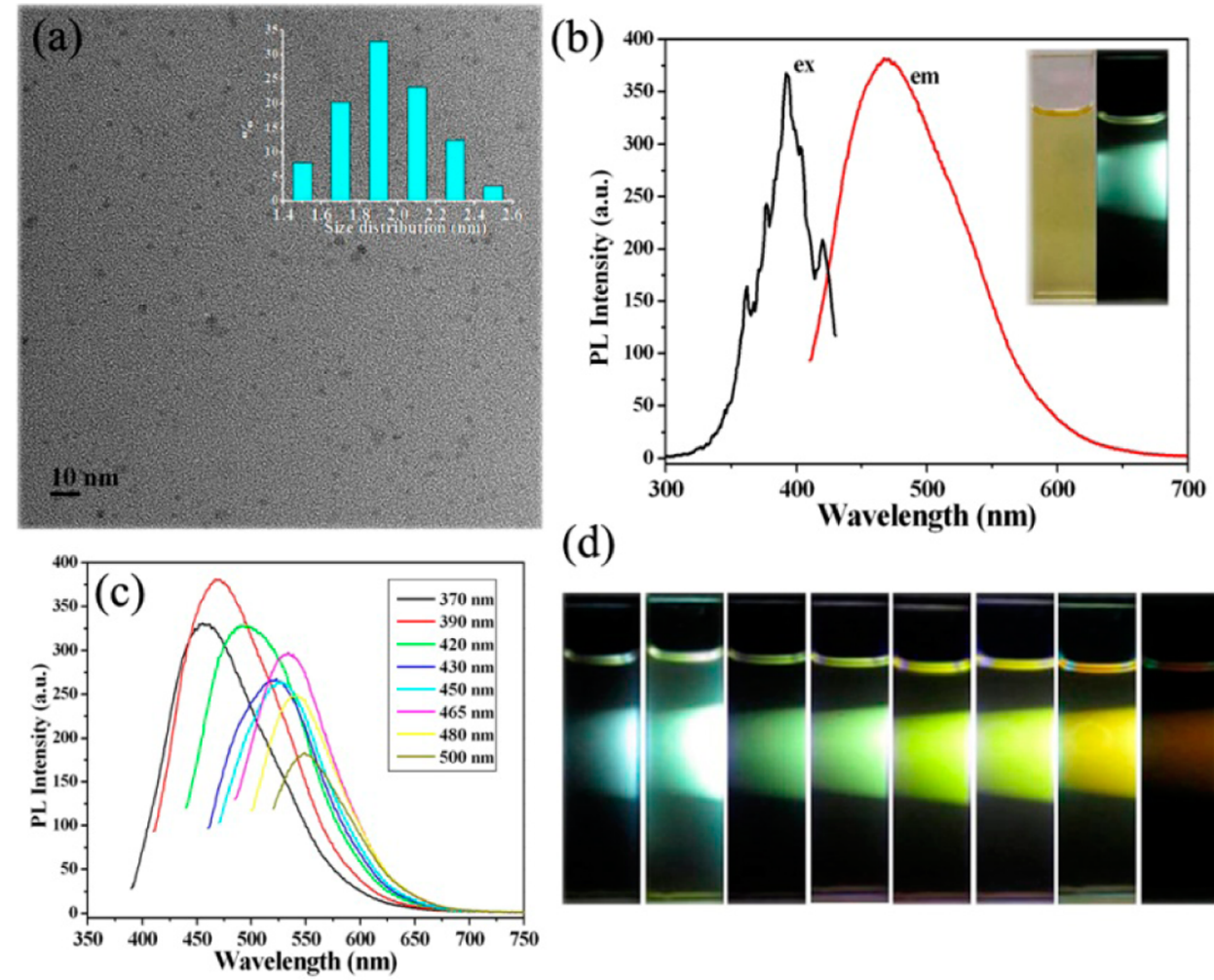

(d)



Figure 1. (a) TEM image of the CDs; (a, inset) size distribution, the average size being $1.9 \pm 0.2 \mathrm{~nm}$; (b) excitation (ex) and emission (em) spectra of the CDs in chloroform solution; ( $b$, inset) photographs of solutions of the fluorescent CDs under (left) room light and (right) UV light illumination; (c) fluorescence spectra of the CDs in chloroform solution with different excitation wavelengths; (d) fluorescence images of the CDs in chloroform solution.

Herein, we report a novel colloidal synthesis of CDs capped by oleic acid (OA) and oleylamine (OLA). These CDs were dispersed in chloroform, exhibiting tunable excitation-dependent emission in the visible light region: by controlling the mole ratio of OLA to OA and thereby modifying the extent of $\mathrm{N}$ doping, $^{39-41}$ the resultant CDs displayed tunable fluorescence with emission wavelengths varying from 430 to $550 \mathrm{~nm}$. With the aid of cysteamine, the resultant CDs were transferred from the organic phase to the aqueous phase. Dual-emission fluorescent nanohybrids were formed by carbodiimide-activated coupling of glutathione-stabilized gold nanoclusters (GSHAuNCs, red PL) and amino-functionalized CDs (blue PL). ${ }^{42-44}$ These novel DEFNs can be used as versatile nanothermometry devices, because their fluorescence intensity ratio (blue/red) correlates strongly with temperature and varies considerably over a wide temperature range $\left(20-75{ }^{\circ} \mathrm{C}\right)$.

\section{EXPERIMENTAL SECTION}

2.1. Materials. Reduced glutathione (GSH, molecular weight of $\left.307 \mathrm{~g} \cdot \mathrm{mol}^{-1}\right)$, 1-octadecene (ODE, 90\%), oleic acid (OA, 90\%), 1ethyl-(3-(3-dimethylamino)propyl)carboxylate hydrochloride (EDC), $\mathrm{N}$-hydroxysuccinimide sodium (NHS), cysteamine and oleylamine (OLA, 70\%) were purchased from Sigma-Aldrich. Citric acid monohydrate (CA), tetrachloroauric(III) acid $\left(\mathrm{HAuCl}_{4}\right)$, chloroform, methanol, isopropanol and acetone were purchased from Sinopharm Chemical Reagent Co., Ltd. (China). All the chemicals were used as received without further purification.

2.2. Synthesis of Organic-Soluble Carbon Dots. The mixture of CA (0.042 g, $20 \mathrm{mmol})$, OA ( $1 \mathrm{~mL}, 2.9 \mathrm{mmol})$, OLA ( $3 \mathrm{~mL}, 6.2$ $\mathrm{mmol})$, and $\mathrm{ODE}(6 \mathrm{~mL}, 17 \mathrm{mmol})$ was degassed at room temperature and heated up to $100{ }^{\circ} \mathrm{C}$ under vacuum for $0.5 \mathrm{~h}$ with stirring. The resultant homogeneous solution was placed under a dry nitrogen atmosphere, heated to $250{ }^{\circ} \mathrm{C}$ slowly and maintained at this temperature for $1 \mathrm{~h}$. The color of the solution became transparent on raising the temperature and finally became dark brown. The temperature of the solution was reduced below $100{ }^{\circ} \mathrm{C}$. The resultant carbon dots were precipitated by addition of acetone, rinsed with methanol, and then redispersed in $20 \mathrm{~mL}$ of chloroform for further use. Using a similar method, other samples with different size were prepared under similar experimental conditions.

2.3. Synthesis of GSH-AuNCs. As per the previously reported method, GSH-AuNCs were prepared through reduction of $\mathrm{HAuCl}_{4}$. $3 \mathrm{H}_{2} \mathrm{O}$ with GSH. ${ }^{6}$ Typically, $\mathrm{HAuCl}_{4}(50 \mathrm{mM}, 0.5 \mathrm{~mL})$ and $\mathrm{GSH}(5$ $\mathrm{mM}, 20 \mathrm{~mL}$ ) were mixed in a $100 \mathrm{~mL}$ round-bottom flask at room temperature. The mixture was stirred at room temperature for $10 \mathrm{~min}$ and was heated at $100{ }^{\circ} \mathrm{C}$ for $6 \mathrm{~h}$. Purification of the resultant GSHAuNCs was effected by centrifuging at $12000 \mathrm{rpm}$ to remove large particles. The supernatants were collected and then dialyzed with deionized water and a dialysis bag (MW cutoff $1000 \mathrm{Da}$ ) to purify the AuNCs from unreacted species. The resultant AuNCs were stored at 4 ${ }^{\circ} \mathrm{C}$ for further use.

2.4. Synthesis of Dual-Emission Fluorescent Nanohybrids. The hydrophobic CDs were further functionalized with thiol compounds, such as cysteamine, which enabled the phase transfer of CDs from hydrophobic to hydrophilic media. ${ }^{38}$ In a typical process, $0.5 \mathrm{~mL}$ of the aforementioned CDs $\left(3.0 \mathrm{mg} \mathrm{mL}^{-1}\right)$ in chloroform was added to $10 \mathrm{~mL}$ of cysteamine solution $\left(5 \mathrm{mg} \mathrm{mL}^{-1}\right)$. A subsequent ultrasonication $(\sim 15 \mathrm{~min})$ led to a stable white emulsion. With vigorous stirring at $60{ }^{\circ} \mathrm{C}$ for $12 \mathrm{~h}$, a transparent solution was formed. After centrifugation to remove the large nanoparticles and dialysis with deionized water to remove free cysteamine, the hydrophilic CDs were collected by freeze-drying.

The dual-emission fluorescent nanohybrids (DEFNs) were built through carbodiimide-activated coupling between GSH-AuNCs and amino-functionalized CDs. In a typical process, $15 \mathrm{mg}$ of EDC and 9 $\mathrm{mg}$ of NHS were added to $2 \mathrm{~mL}$ of PBS buffer ( $\mathrm{pH}$ 6.86) containing 
$10 \mathrm{mg}$ of GSH-AuNCs. The mixture was treated by sonication in the dark at room temperature for $30 \mathrm{~min}$, and then mixed with $2 \mathrm{~mL}$ of an aqueous solution containing $20 \mathrm{mg}$ of amino-functionalized CDs. This solution was allowed to react under magnetic stirring for $8 \mathrm{~h}$. Finally, the resultant nanohybrids were precipitated by the addition of $20 \mathrm{~mL}$ of isopropyl alcohol, collected through centrifugation at $12000 \mathrm{rpm}$, and then redispersed in $10 \mathrm{~mL}$ of aqueous solution for further application.

2.5. Temperature Measurement of Living Cells. First, 293T cells were grown on a circular coverslip (diameter $18 \mathrm{~mm}$ ) overnight at $37{ }^{\circ} \mathrm{C}$ and $5 \% \mathrm{CO}_{2}$. Subsequently, the cells were incubated with 10 $\mu \mathrm{g} \mathrm{mL}^{-1}$ DEFNs in serum-free DMEM for $2 \mathrm{~h}$, washed with PBS and fixed with $1 \mathrm{~mL}$ of $4 \%$ (by mass) paraformaldehyde in PBS for $15 \mathrm{~min}$. The cells were then washed with warm PBS to remove the excess DEFNs. Prior to inspection with a confocal fluorescence microscope, the sample temperature was adjusted using a heater that controlled the in situ temperature of cells during the microscopy observation. For each point, the temperature was set and equilibrated for $5 \mathrm{~min}$ before the spectral measurements. The confocal microscopy images were taken using an Olympus Fluoview FV1000.

2.6. Characterization. Photoluminescence experiments were performed with a Shimadzu RF-5301 PC spectrofluorimeter (Shimadzu, Japan). UV-vis absorption spectra were obtained using a TU-1991 UV-vis spectrophotometer. X-ray photoelectron spectroscopy (XPS) with $\mathrm{Mg} \mathrm{K} \alpha$ excitation $(1253.6 \mathrm{eV})$ was collected using a VG ES-CALAB MKII spectrometer (ThermoFisher Scientific, USA); the binding energy was calibrated with $\mathrm{C} 1 \mathrm{~s}$ at $284.6 \mathrm{eV}$. Fourier transform infrared spectroscopy (FTIR) was measured in the range of $500-4000 \mathrm{~cm}^{-1}$ using a Nicolet 6700 FTIR spectrophotometer. Transmission electron microscopy analyses to study the morphology and mean diameter of the resultant samples were carried out on a JEM-2100F (FEI, USA), operating at an accelerating voltage of $200 \mathrm{kV}$. Dynamic light scattering (DLS) and Zeta potential measurements were performed using a Zetasizer NanoZS (Malvern Instruments). The confocal microscopy images were observed under a confocal fluorescence microscope (FV1000, Olympus).

\section{RESULTS AND DISCUSSION}

The carbon dots (CDs) were prepared by the colloidal synthesis method described in the Experimental Section. Citric acid (CA) was adopted as the $\mathrm{C}$ source and was carbonized at high temperature. The resultant $\mathrm{CDs}$ were stabilized in situ by OA and OLA. The morphology and structure of the CDs were confirmed by various analyses. Figure la shows a transmission electron microscopy (TEM) image of the CDs, revealing that the CDs are distributed without apparent aggregation, and that the diameters of the particles are ca. $1.5-2.5 \mathrm{~nm}$, with the average value being $1.9 \pm 0.2 \mathrm{~nm}$ (inset of Figure 1a). The surface groups were also investigated by X-ray photoelectron spectroscopy (XPS) (Figure S1). A high-resolution XPS spectrum in the $\mathrm{C}$ 1s window (Figure $\mathrm{S} 1$ ) revealed the presence of $\mathrm{C}-\mathrm{C}$ or $\mathrm{C}=\mathrm{C}, \mathrm{C}=\mathrm{O}$, and $\mathrm{C}-\mathrm{N}$ bonds, which mostly stem from carbogenic cores, surface carbonyl groups, and surface-bound oleylamine molecules, respectively. ${ }^{39,45}$ In chloroform, the optimal excitation and emission wavelengths of the CDs are 390 and $470 \mathrm{~nm}$, respectively (Figure 1b). A diluted solution of the as-prepared CDs in chloroform appeared pale yellow under visible light (inset of Figure 1b), while emitting blue-green fluorescence under UV light illumination (inset of Figure 1b). The as-prepared CDs in the present studies also exhibited excitation-dependent emission behavior without an accompanying significant decrease in fluorescence emission intensity, due to the size distribution $(1.5-2.5 \mathrm{~nm}$ ) and the novel colloidal method. As shown in Figure 1c, when the CDs were excited at wavelengths progressing from 370 to $500 \mathrm{~nm}$, the corresponding emission peaks shifted concom- itantly from 456 to $550 \mathrm{~nm}$ while maintaining comparable intensities. The strongest photoluminescence (PL) peak appeared at $470 \mathrm{~nm}$ upon $390 \mathrm{~nm}$ excitation, which is consistent with the photoluminescence excitation (PLE) and PL spectra shown in Figure 1b. The as-prepared CDs exhibited selective emission across a broad color range from blue to orange (Figure 1c,d); such excitation-dependent PL behavior is common for fluorescent CDs and useful in multicolor imaging applications. ${ }^{46-50}$

The PL properties of CDs can be influenced by quantum size effects, surface states, zigzag sites, and so on. ${ }^{51-53}$ Previous reports had ascertained that CDs possessing tunable emission wavelengths could be prepared by adjusting the extent of N, S or $\mathrm{P}$ doping. ${ }^{43}$ In the present study, we modified the ratios of OA/OLA to vary the $\mathrm{N}$ amount on the surface of the resultant CDs. As shown in Figure S2, upon increasing the amount of OLA, the room temperature PL emission exhibited a significant red shift, from 430 to $550 \mathrm{~nm}$. The emission wavelengths of the samples span blue, cyan, and yellow, with the emission sufficiently bright that it can be readily detected with the naked eye (inset of Figure S2). TEM images are shown in Figure S3, demonstrating that the as-prepared CDs were welldispersed, and with an average size that decreased with increasing content of OLA, and thereby $\mathrm{N}$ content, the latter being confirmed by XPS (Figure S4); the extent of $\mathrm{N}$ doping correlates with the redshift of the emission of the CDs (i.e., the fluorescence is tunable). ${ }^{51-53}$

The organic solvent-soluble CDs can be easily transferred to the aqueous phase by using thiol compounds, such as cysteamine. $^{39-41}$ In aqueous solution, the cysteamine-CDs were well dispersed, emitting blue light (Figure S5). Fourier transform infrared (FTIR) spectroscopy (Figure S6) was employed to identify the functional groups present on the surfaces of the CDs in aqueous solution; while the intensity of the characteristic $\mathrm{S}-\mathrm{H}$ stretching band decreased, the bands characteristic of the other functional groups of cysteamine were still present, supporting the proposed formation of aminofunctionalized CDs (Figure S6).

Glutathione-stabilized gold nanoclusters (GSH-AuNCs) were then prepared, displaying red fluorescence centered at $605 \mathrm{~nm}$. The as-prepared GSH-AuNCs were well-dispersed in aqueous solution, with an average size of ca. $2.4 \mathrm{~nm}$ (Figure S7) ${ }^{54}$ due to the presence of GSH on the surface of the AuNCs, the surface modification being beneficial for subsequent applications. ${ }^{55-57}$ Our DEFN-based temperature sensor was then assembled through carbodiimide-activated coupling of the GSH-AuNCs and the amino-functionalized CDs (Figure 2a). ${ }^{42,43}$ A typical TEM image (Figure 2b) revealed peanut-like and monodisperse nanohybrids. The average size of the nanohybrids was ca. $5 \mathrm{~nm}$, which is consistent with CD-AuNC addition. The CD and AuNC content in the peanut-like nanostructure was clearly seen by high resolution TEM (HRTEM; inset of Figure $2 \mathrm{~b}$ ). Dynamic light scattering measurements confirmed the size of the DEFNs, with a number-averaged diameter of $\sim 4.8 \mathrm{~nm}$, which is in agreement with the results from TEM (Figure S8). As shown in Figure S9, the maximum absorption peaks of the CDs and AuNCs are in the UV region. Therefore, a $365 \mathrm{~nm}$ wavelength laser could excite both materials in the hybrid sensor, and indeed under $365 \mathrm{~nm}$ excitation, the nanohybrid system exhibited two well-resolved emission peaks at 430 and $605 \mathrm{~nm}$ (Figure 2c). There is a narrow overlap between the absorption spectrum of the AuNCs and the PL spectrum of the 

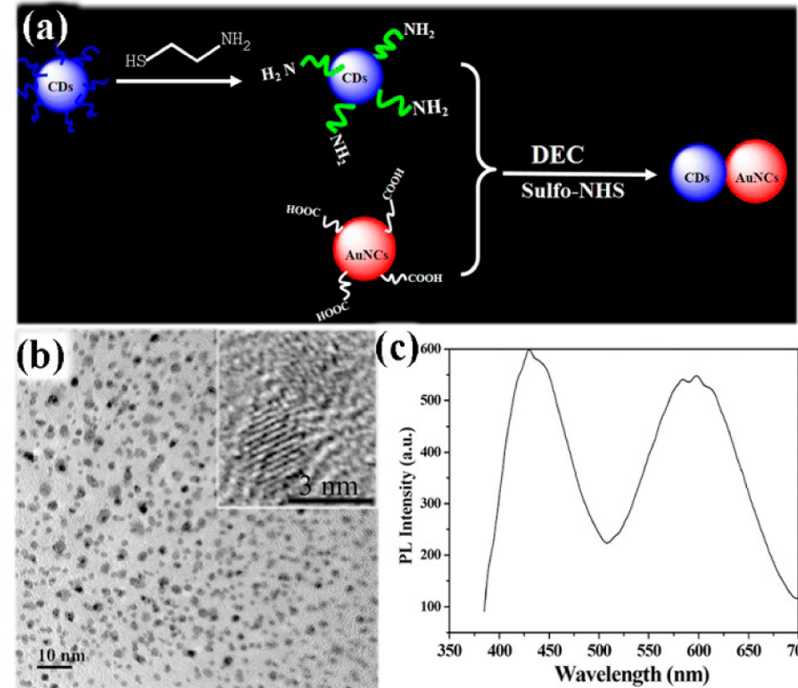

(d)
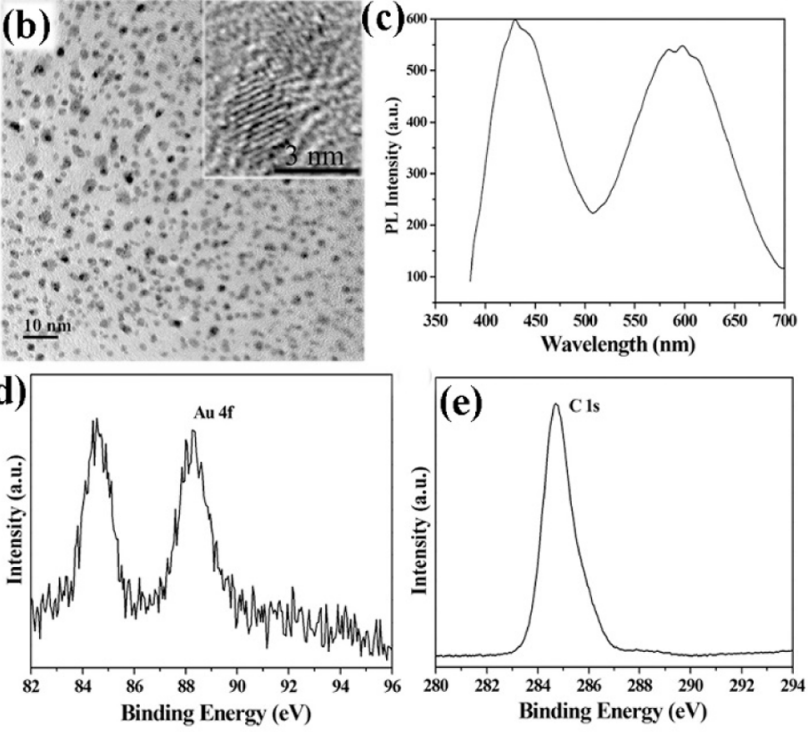

Figure 2. (a) Schematic illustration of the synthesis of the DEFNs from CDs and GSH-AuNCs; (b) TEM and (inset) HRTEM images of the DEFNs; (c) fluorescence spectrum of the DEFNs in aqueous solution; (d) Au 4f XPS spectrum of the DEFNs; and (e) C 1s XPS spectrum of the DEFNs.

CDs, but in the overlap range, the $\mathrm{PL}$ of the CDs was rather weak. Moreover, the PL quantum yields (QYs) of the AuNCs and the $\mathrm{CDs}$ were found to be $5.5 \%$ and $3.8 \%$ using quinine sulfate $\left(0.1 \mathrm{M} \mathrm{H}_{2} \mathrm{SO}_{4}\right.$ as solvent; $\left.\mathrm{QY}=0.54\right)$ as a reference. After forming the DEFNs, the QYs were 5.8\% (AuNCs) and $3.3 \%$ (CDs), respectively, so the energy transfer between the AuNCs and the CDs in the DEFNs was trivial. The elemental composition of the nanohybrids was confirmed by XPS. The $\mathrm{Au}$ $4 \mathrm{f}$ binding energies ( 88.1 and $84.3 \mathrm{eV}$; Figure 2d) are consistent with the electronic structure of AuNCs, ${ }^{58,59}$ while the $\mathrm{C}$ 1s binding energy matched those of previously reported CDs (Figure 2e). ${ }^{41,45}$ The purity of the DEFNs was confirmed by gel electrophoresis (Figure S10). It was possible to track the movement of the nanomaterials along the gel due to their different Zeta potentials, particle size and so on. The GSHAuNCs did not move at all since their Zeta potential was about $0 \mathrm{mV}$ (Figure S10a). Instead, the movement of $\mathrm{NH}_{2}-\mathrm{CDs}$ (belt b) and DEFNs (belt a) were observed under UV light. The speed of the DEFNs (belt a) was less than that of the $\mathrm{NH}_{2}-\mathrm{CDs}$ (belt b) along the gel, which was consistent with the results of Zeta potentials (Figure S10). The bright and narrow signal of the DEFNs (belt a in lane 3) clearly indicated that the nanohybrids were highly pure and uniform, while only a weak $\mathrm{NH}_{2}-\mathrm{CDs}$ signal (belt $\mathrm{b}$ in lane 3) was observed (Figure $\mathrm{S} 10 \mathrm{~b})$. The CD-AuNCs therefore preserved the composition and structure of the constituent CDs and AuNCs, confirming the successful synthesis of the dual-emission nanohybrids.

To explore the potential applications of the DEFNs in intracellular nanothermometry, we investigated their temper- ature-dependent response. The PL spectra of the DEFNs were measured at temperatures ranging from 20 to $75{ }^{\circ} \mathrm{C}$. The measurements were conducted with both increasing and decreasing temperature to ensure reproducibility, the resulting PL spectra being shown in Figure 3a and Figure S11a. GSHAuNCs have been used as nanothermometry devices because of the temperature sensitivity of their fluorescence lifetime and emission intensity; ${ }^{6}$ the nonradiative recombination of electrons and holes increases as the temperature increases, whereas the radiative transition rate remains constant, reducing the fluorescence intensity corresponding to emission from the excited state. ${ }^{13,60}$ Thus, upon increasing the temperature, we observed a concomitant decrease in intensity of red emission from the GSH-AuNCs, while the blue emission from the CDs changed little (Figure $3 \mathrm{a}$ and Figure S11a). Although the fluorescence of the CDs in the DEFNs differed little with temperature change, the fluorescence of the AuNCs decreased rapidly with increasing temperature. A linear relationship was observed between the ratios of emission intensities at the two emission wavelengths $\left(I_{430} / I_{605}\right)$ and the temperature (Figure $3 \mathrm{~b})$. Thus, the varying fluorescence of the CDs with temperature changes did not affect the efficient function of the DEFNs as a ratiometric temperature sensor. Unlike semiconductor QDs, ${ }^{10,61}$ which display remarkable temperature-dependent spectral shifts, the emission spectra of CDs and NCs do not shift within the investigated temperature window. The changes in the intensities of the two emission peaks resulted in a continuous fluorescence color variation (inset of Figure $3 \mathrm{~b}$ ). The fluorescence at $605 \mathrm{~nm}$ decreased with increasing temperature, and was accompanied by a distinguishable fluorescence color change from red to violet. To obtain sensitivity information, the blue bands at $430 \mathrm{~nm}$ were normalized, the resultant PL spectra being shown in Figure S12. For the red band, the PL intensity decreased by $99.0 \%$ with a temperature variation of $75{ }^{\circ} \mathrm{C}$, indicating a sensitivity of approximately $1.8 \%{ }^{\circ} \mathrm{C}^{1-}$, which is competitive with previous reports. $9,21,62-64$ The fluorescent nanosensor therefore functioned as an ideal ratiometric temperature sensor with high reliability and accuracy. A Commission Internationale de L'Eclairage (CIE) 1931 chromaticity diagram in the temperature range of $20-70{ }^{\circ} \mathrm{C}$ showed that the system color shifts between red and violet (Figure 3c), consistent with the PL spectra of Figure 3a. The reproducibility of the emission intensity measurements at the different temperatures indicated that the DEFNs have excellent stability over the temperature range. The luminescence switching operations were repeated for six consecutive heating and cooling cycles between 20 and $60{ }^{\circ} \mathrm{C}$ (Figure 3d; see corresponding spectra in Figure S13. This fatigue-resistant performance suggests that the DEFNs may be applicable as PL thermometers and biosensors in cell monitoring.

As has been previous reported, the fluorescence of CDs and GSH-AuNCs show high stability. ${ }^{11,58}$ After forming the DEFNs, the dual fluorescence and the fluorescent intensity ratios did not change over the entire physiologically relevant $\mathrm{pH}$ range of 4-9 (Figure S14a), in the presence of the physiological ionic strength (Figure S14b), in the presence of biomolecules (Figure S14c) and after exposing the DEFNs to a $500 \mathrm{~W}$ Xe lamp for $150 \mathrm{~min}$ (Figure S14d). Therefore, the asprepared DEFNs from the present study possess high stability and are particular appropriate for practical applications. This dual-emission fluorescent temperature sensor was also assayed in $10 \mathrm{mM}$ phosphate-buffered saline (PBS) solution ( $\mathrm{pH} 7.4$, 
(a)



(c)

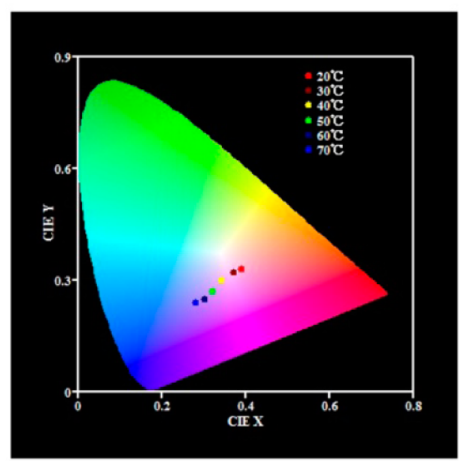

(b)

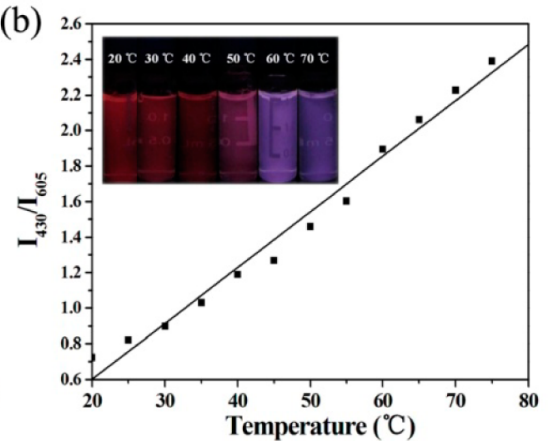

(d)



Figure 3. (a) Fluorescence spectra (excitation $367 \mathrm{~nm}$ ) of DEFNs with an increase of temperature from 20 to $75{ }^{\circ} \mathrm{C}$ (top to bottom); (b) the ratios of the emission intensities at 430 and $605 \mathrm{~nm}\left(I_{430} / I_{605}\right)$ versus temperature; (inset) photographs of DEFNs at various temperatures under a UV lamp with an excitation wavelength at $365 \mathrm{~nm}$; (c) CIE chromaticity diagram showing the temperature dependence of the $(x, y)$ color coordinates of the DEFNs; (d) temperature-induced switching of the emission intensities ratio of the DEFNs (alternating between 20 and $60{ }^{\circ} \mathrm{C}$ ).

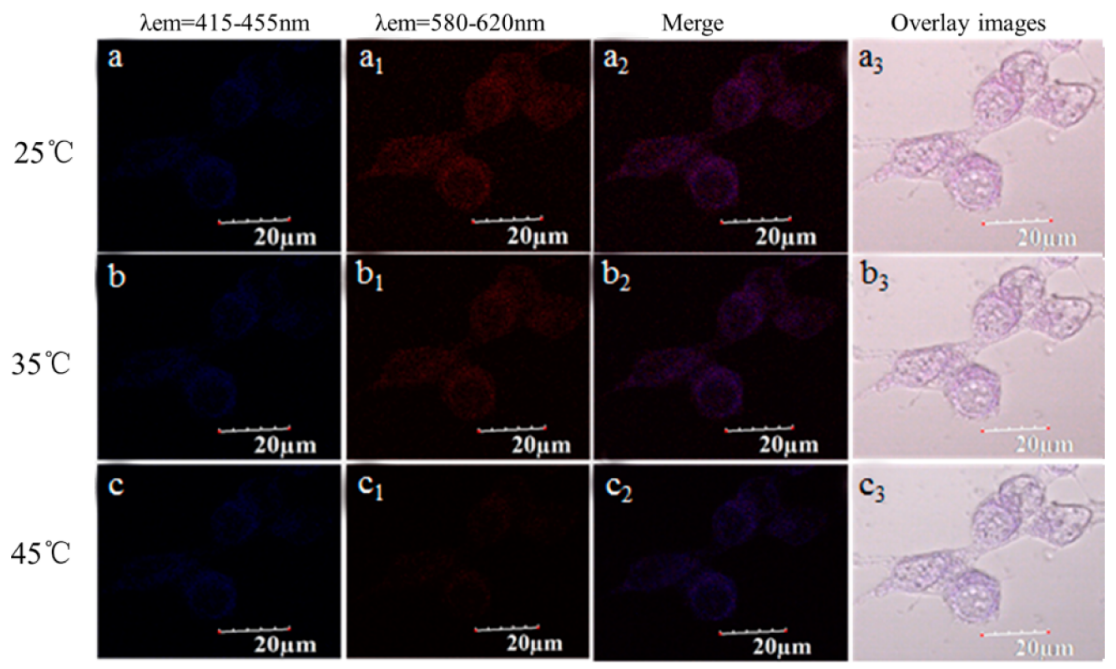

Figure 4. Confocal fluorescence microscopy images of $293 \mathrm{~T}$ cells with internalized DEFNs at three different temperatures: (a) 25, (b) 35 , and (c) 45 ${ }^{\circ} \mathrm{C}$. Fluorescence images at (1) the blue channel and (2) the red channel; (3) overlap of the fluorescence images; and (4) corresponding bright field images.

Figure S15a), with the ratios of fluorescence intensities showing a linear relationship with increasing temperature over the range 20 to $45{ }^{\circ} \mathrm{C}$ (Figure S15b). This temperature range is larger than the physiological temperature range, suggesting that the DEFNs have considerable potential for in vivo temperature sensing applications. Toxicity is a major factor that needs consideration in the design of an intracellular sensor system for subsequent biological applications. To evaluate the cytotoxicity of the DEFNs, we conducted a thiazolyl blue tetrazolium bromide (MTT) assay on cells loaded with DEFNs. ${ }^{65,66}$ As shown in Figure S16, the viability of the 293 T cells remained above $80 \%$ following incubation with the DEFNs, even at a concentration of $100 \mu \mathrm{g} / \mathrm{mL}$ for $24 \mathrm{~h}$, considerably more forcing conditions than those used for cell incubation and imaging. The results confirm that the as-prepared nanohybrids are biocompatible and have low toxicity and can therefore be used in optical thermometry in living cells.

The utility of the DEFN thermometers in monitoring intracellular temperature was investigated using confocal fluorescence microscopy; 293T cells were selected as a model system, and three samples under different environmental temperatures were tested, as shown in Figure 4. After 
incubating with the DEFNs, the 293T cells maintained their normal morphology, consistent with good biocompatibility of the DEFNs. The blue and red channels both displayed fluorescence signals with typical cytosolic colocalization. The DEFNs were therefore delivered rapidly into the cytoplasm and maintained their dual-emission characteristics. Upon increasing the temperature, the fluorescence emission color of the probe changed from red (Figure $4 a_{3}$ ) to violet (Figure $4 c_{3}$ ), consistent with the changes in the fluorescence spectra observed in aqueous solution and PBS solution. These changes were clearly observed in the separate channels in Figure 4; upon increasing the temperature, the red fluorescence image from the AuNCs became dimmer whereas the blue reference channel showed almost no change. The results confirmed the potential of carbon-dot-based dual emission nanohybrid systems in sensing temperature profiles at the subcellular level and, more broadly, highlight their potential in fundamental biology research.

\section{CONCLUSION}

In summary, fluorescent CDs were prepared in the organic phase. These CDs exhibited excitation-dependent emission behavior, with PL peaks varying from 456 to $550 \mathrm{~nm}$, and $\mathrm{N}$ doping-dependent tunable PL emission in the range of 430$550 \mathrm{~nm}$. Carbon-dot-based DEFNs were fabricated through condensation between carbodiimide-activated GSH-AuNCs and amino-functionalized CDs. The DEFNs displayed ideal single-excitation, dual-emission behavior with two wellresolved, intensity-comparable fluorescence peaks. These features render the DEFNs very attractive due to their capacity to enhance imaging contrast and accuracy. The DEFNs are sensitive sensors for quantitative ratiometric detection of external environmental temperature with good reproducibility. The DEFNs were also employed to monitor intracellular temperature differences $\left(25-45^{\circ} \mathrm{C}\right)$ in living cells. Because of their facile synthesis, attractive fluorescence properties, and excellent biocompatibility, the DEFNs are promising candidates for cellular imaging and temperature sensing at the molecular level.

\section{ASSOCIATED CONTENT}

\section{S Supporting Information}

The Supporting Information is available free of charge on the ACS Publications website at DOI: 10.1021/acsami.5b11317.

Additional emission spectra, FTIR spectra, XPS analysis results, UV-vis spectra, TEM images, DLS, Zeta potentials, and MTT results. (PDF)

\section{AUTHOR INFORMATION}

\section{Corresponding Author}

*E-mail: chi.zhang@jiangnan.edu.cn and chi.zhang@anu.edu. au. Fax: +86-510-85917763.

\section{Notes}

The authors declare no competing financial interest.

\section{ACKNOWLEDGMENTS}

This work was supported by the National Natural Science Foundation of China (Nos. 50925207, 51432006, and 51503085), the Ministry of Science and Technology of China for the International Science Linkages Program (No. 2011DFG52970), the Natural Science Foundation of Jiangsu Province, China (No. BK20140157), the Changjiang Innovation Research Team (IRT14R23), the Programme of
Introducing Talents of Discipline to Universities (111 Project B13025). M.G.H. and C.Z. thank the Australian Research Council (ARC) for support.

\section{REFERENCES}

(1) Kucsko, G.; Maurer, P. C.; Yao, N. Y.; Kubo, M.; Noh, H. J.; Lo, P. K.; Park, H.; Lukin, M. D. Nanometre-Scale Thermometry in a Living Cell. Nature 2013, 500, 54-58.

(2) Feng, J.; Tian, K.; Hu, D. H.; Wang, S. Q.; Li, S. Y.; Zeng, Y.; Li, Y.; Yang, G. A Triarylboron-Based Fluorescent Thermometer: Sensitive Over a Wide Temperature Range. Angew. Chem., Int. Ed. 2011, 50, 8072-8076.

(3) Mahmoudi, M.; Abdelmonem, A. M.; Behzadi, S.; Clement, J. H.; Dutz, S.; Ejtehadi, M. R.; Hartmann, R; Kantner, K.; Linne, U.; Maffre, P. Temperature: The "Ignored" Factor at the NanoBio Interface. ACS Nano 2013, 7, 6555-6562.

(4) Li, Q.; He, Y.; Chang, J.; Wang, L.; Chen, H. Z.; Tan, Y. W.; Wang, H. Y.; Shao, Z. Z. Surface-Modified Silicon Nanoparticles with Ultrabright Photoluminescence and Single-Exponential Decay for Nanoscale Fluorescence Lifetime Imaging of Temperature. J. Am. Chem. Soc. 2013, 135, 14924-14927.

(5) Okabe, K.; Inada, N.; Gota, C.; Harada, Y.; Funatsu, T.; Uchiyama, S. Intracellular Temperature Mapping with a Fluorescent Polymeric Thermometer and Fluorescence Lifetime Imaging Microscopy. Nat. Commun. 2012, 3, 705-713.

(6) Shang, L.; Stockmar, F.; Azadfar, N.; Nienhaus, G. U. Intracellular Thermometry by Using Fluorescent Gold Nanoclusters. Angew. Chem., Int. Ed. 2013, 52, 11154-11157.

(7) Zhou, D.; Zhang, H. Critical Growth Temperature of Aqueous CdTe Quantum Dots is Non-Negligible for Their Application as Nanothermometers. Small 2013, 9, 3195-3197.

(8) Cauzzi, D.; Pattacini, R.; Delferro, M.; Dini, F.; Di Natale, C. D.; Paolesse, R.; Bonacchi, S.; Montalti, M.; Zaccheroni, N.; Calvaresi, M. Temperature-Dependent Fluorescence of $\mathrm{Cu}_{5}$ Metal Clusters: A Molecular Thermometer. Angew. Chem., Int. Ed. 2012, 51, 9662-9665.

(9) Vetrone, F.; Naccache, R.; Zamarron, A.; Juarranz de la Fuente, A.; Sanz-Rodríguez, F.; Martinez Maestro, L.; Martín Rodriguez, E.; Jaque, D.; García Solé, J.; Capobianco, J. A. Temperature Sensing Using Fluorescent Nanothermometers. ACS Nano 2010, 4, 32543258.

(10) Zhou, D.; Lin, M.; Liu, X.; Li, J.; Chen, Z. L.; Yao, D. Z.; Sun, H. C.; Zhang, H.; Yang, B. Conducting the Temperature-Dependent Conformational Change of Macrocyclic Compounds to the Lattice Dilation of Quantum Dots for Achieving an Ultrasensitive Nanothermometer. ACS Nano 2013, 7, 2273-2283.

(11) Wang, C. X.; Xu, Z. Z.; Cheng, H.; Lin, H. H.; Humphrey, M. G.; Zhang, C. A Hydrothermal Route to Water-Stable Luminescent Carbon Dots as Nanosensors for $\mathrm{pH}$ and Temperature. Carbon 2015, $82,87-95$.

(12) Brites, C. D.; Lima, P. P.; Silva, N. J.; Millán, A.; Amaral, V. S.; Palacio, F.; Carlos, L. D. Ratiometric Highly Sensitive Luminescent Nanothermometers Working in the Room Temperature Range: Applications to Heat Propagation in Nanofluids. Nanoscale 2013, 5, $7572-7580$

(13) Wang, C.; Ling, L.; Yao, Y. G.; Song, Q. J. One-Step Synthesis of Fluorescent Smart Thermoresponsive Copper Clusters: A Potential Nanothermometer in Living Cells. Nano Res. 2015, 8, 1975-1986.

(14) Löw, P.; Kim, B.; Takama, N.; Bergaud, C. High-SpatialResolution Surface-Temperature Mapping Using Fluorescent Thermometry. Small 2008, 4, 908-914.

(15) Han, B.; Hanson, W. L.; Bensalah, K.; Tuncel, A.; Stern, J. M.; Cadeddu, J. A. Development of Quantum Dot-Mediated Fluorescence Thermometry for Thermal Therapies. Ann. Biomed. Eng. 2009, 37, 1230-1239.

(16) Zhu, A. W.; Qu, Q.; Shao, X. L.; Kong, B.; Tian, Y. Carbon-DotBased Dual-Emission Nanohybrid Produces a Ratiometric Fluorescent Sensor for In Vivo Imaging of Cellular Copper Ions. Angew. Chem., Int. Ed. 2012, 51, 7185-7189. 
(17) Chen, T. T.; Hu, Y. H.; Cen, Y.; Chu, X.; Lu, Y. A DualEmission Fluorescent Nanocomplex of Gold-Cluster-Decorated Silica Particles for Live Cell Imaging of Highly Reactive Oxygen Species. J. Am. Chem. Soc. 2013, 135, 11595-11602.

(18) McLaurin, E. J.; Bradshaw, L. R.; Gamelin, D. R. Dual-Emitting Nanoscale Temperature Sensors. Chem. Mater. 2013, 25, 1283-1292.

(19) Bayles, A. R.; Chahal, H. S. D. S.; Chahal, C. P.; Goldbeck, B. E.; Cohen, B. A. Helms, Rapid Cytosolic Delivery of Luminescent Nanocrystals in Live Cells with Endosome-Disrupting Polymer Colloids. Nano Lett. 2010, 10, 4086-4092.

(20) Wang, S. P.; Westcott, S.; Chen, W. Nanoparticle Luminescence Thermometry. J. Phys. Chem. B 2002, 106, 11203-11209.

(21) Ye, F.; Wu, C. F.; Jin, Y. H.; Chan, Y.-H.; Zhang, X. J.; Chiu, D. T. Ratiometric Temperature Sensing with Semiconducting Polymer Dots. J. Am. Chem. Soc. 2011, 133, 8146-8149.

(22) Ding, C. Q.; Zhu, A. W.; Tian, Y. Functional Surface Engineering of C-Dots for Fluorescent Biosensing and in Vivo Bioimaging. Acc. Chem. Res. 2014, 47, 20-30.

(23) Wang, X.; Cao, L.; Yang, S. T.; Lu, F.; Meziani, M. J.; Tian, L. L.; Sun, K. W.; Bloodgood, M. A.; Sun, Y. P. Bandgap-Like Strong Fluorescence in Functionalized Carbon Nanoparticles. Angew. Chem., Int. Ed. 2010, 49, 5310-5314.

(24) Song, Y. B.; Zhu, S. J.; Yang, B. Bioimaging Based on Fluorescent Carbon Dots. RSC Adv. 2014, 4, 27184-27200.

(25) Fang, Y. X.; Guo, S. J.; Li, D.; Zhu, C. Z.; Ren, W.; Dong, S. J.; Wang, E. K. Easy Synthesis and Imaging Applications of Cross-Linked Green Fluorescent Hollow Carbon Nanoparticles. ACS Nano 2011, 6, 400-409.

(26) Zhu, S. J.; Meng, Q. N.; Wang, L.; Zhang, J. H.; Song, Y. B.; Jin, H.; Zhang, K.; Sun, H. C.; Wang, H. Y.; Yang, B. Highly Photoluminescent Carbon Dots for Multicolor Patterning, Sensors, and Bioimaging. Angew. Chem., Int. Ed. 2013, 52, 3953-3957.

(27) Nie, H.; Li, M. J.; Li, Q. S.; Liang, S. J.; Tan, Y. Y.; Sheng, L.; Shi, W.; Zhang, S. X. A. Carbon Dots with Continuously Tunable FullColor Emission and Their Application in Ratiometric $\mathrm{pH}$ Sensing. Chem. Mater. 2014, 26, 3104-3112.

(28) Li, H. T.; He, X. D.; Kang, Z. H.; Huang, H.; Liu, Y.; Liu, J. L.; Lian, S. Y.; Tsang, C. H. A.; Yang, X. B.; Lee, S. T. Water-Soluble Fluorescent Carbon Quantum Dots and Photocatalyst Design. Angew. Chem., Int. Ed. 2010, 49, 4430-4434.

(29) Lou, Q.; Qu, S. N.; Jing, P. T.; Ji, W. Y.; Li, D.; Cao, J. S.; Zhang, H.; Liu, L.; Zhao, J. L.; Shen, D. Z. Water-Triggered Luminescent "Nano-Bombs" Based on Supra-(Carbon Nanodots). Adv. Mater. 2015, 27, 1389-1394.

(30) Zhou, L.; He, B. Z.; Huang, J. C. Amphibious Fluorescent Carbon Dots: One-Step Green Synthesis and Application for Lightemitting Polymer Nanocomposites. Chem. Commun. 2013, 49, 80788080.

(31) Lai, T. T.; Zheng, E. H.; Chen, L. X.; Wang, X. Y.; Kong, L. C.; You, C. P.; Ruan, Y. M.; Weng, X. X. Hybrid Carbon Source for Producing Nitrogen-Doped Polymer Nanodots: One-pot Hydrothermal Synthesis, Fluorescence Enhancement and Highly Selective Detection of Fe (III). Nanoscale 2013, 5, 8015-8021.

(32) Lan, M. H.; Zhang, J. F.; Chui, Y.-S.; Wang, P. F.; Chen, X. F.; Lee, C.-S.; Kwong, H.-L.; Zhang, W. J. Carbon Nanoparticle-Based Ratiometric Fluorescent Sensor for Detecting Mercury Ions in Aqueous Media and Living Cells. ACS Appl. Mater. Interfaces 2014, 6, 21270-21278.

(33) Ju, E. G.; Liu, Z.; Du, Y. P.; Tao, Y.; Ren, J. S.; Qu, X. G. Heterogeneous Assembled Nanocomplexes for Ratiometric Detection of Highly Reactive Oxygen Species in vitro and in vivo. ACS Nano 2014, 8, 6014-6023.

(34) Zhao, P.; He, K. Y.; Han, Y. T.; Zhang, Z.; Yu, M. Z.; Wang, H. H.; Huang, Y.; Nie, Z.; Yao, S. Z. Near-Infrared Dual-Emission Quantum Dots-Gold Nanoclusters Nanohybrid via Co-Template Synthesis for Ratiometric Fluorescent Detection and Bioimaging of Ascorbic Acid in vitro and in vivo. Anal. Chem. 2015, 87, 9998-10005.

(35) Shen, L. M.; Zhang, L. P.; Chen, M. L.; Chen, X. W.; Wang, J. $\mathrm{H}$. The Production of $\mathrm{pH}$-sensitive Photoluminescent Carbon
Nanoparticles by the Carbonization of Polyethylenimine and Their Use for Bioimaging. Carbon 2013, 55, 343-349.

(36) Wang, W.; Li, Y. M.; Cheng, L.; Cao, Z. Q.; Liu, W. G. Watersoluble and Phosphorus-containing Carbon Dots with Strong Green Fluorescence for Cell Labeling. J. Mater. Chem. B 2014, 2, 46-48.

(37) Qu, S. N.; Shen, D. Z.; Liu, X. Y.; Jing, P. T.; Zhang, L. G.; Ji, W. Y.; Zhao, H. F.; Fan, X. W.; Zhang, H. Highly Luminescent CarbonNanoparticle-Based Materials: Factors Influencing Photoluminescence Quantum Yield. Part. Part. Syst. Charact. 2014, 31, 1175-1182.

(38) Pan, D. Y.; Zhang, J. C.; Li, Z.; Wu, C.; Yan, X.; Wu, M. H. Observation of $\mathrm{pH}-$, Solvent-, Spin-, and Excitation-Dependent Blue Photoluminescence from Carbon Nanoparticles. Chem. Commun. 2010, 46, 3681-3683.

(39) Kwon, W.; Lee, G.; Do, S.; Joo, T.; Rhee, S. W. Size-Controlled Soft-Template Synthesis of Carbon Nanodots Toward Versatile Photoactive Materials. Small 2014, 10, 506-513.

(40) Chan, W. C. W.; Nie, S. M. Quantum Dot Bioconjugates for Ultrasensitive Nonisotopic Detection. Science 1998, 281, 2016-2018.

(41) Xie, R. G.; Kolb, U.; Li, J.; Basche, T.; Mews, A. Synthesis and Characterization of Highly Luminescent CdSe-Core CdS $/ \mathrm{Zn}_{0.5} \mathrm{Cd}_{0.5} \mathrm{~S} /$ ZnS Multishell Nanocrystals. J. Am. Chem. Soc. 2005, 127, 7480-7488.

(42) Fernandez, B.; Galvez, N.; Cuesta, R.; Hungria, A. B.; Calvino, J. J.; Domínguez-Vera, J. M. Quantum Dots Decorated with Magnetic Bionanoparticles. Adv. Funct. Mater. 2008, 18, 3931-3935.

(43) Cheng, H.; Wang, C. X.; Xu, Z. Z.; Lin, H. H.; Zhang, C. Gold Nanoparticle-Enhanced Near Infrared Fluorescent Nanocomposites for Targeted Bio-Imaging. RSC Adv. 2015, 5, 20-26.

(44) Zhang, Y. Q.; Ma, D. K.; Zhuang, Y.; Zhang, X.; Chen, W.; Hong, L. L.; Yan, Q. X.; Yu, K.; Huang, S. M. One-Pot Synthesis of Ndoped Carbon Dots with Tunable Luminescence Properties. J. Mater. Chem. 2012, 22, 16714-16718.

(45) Zhai, X. Y.; Zhang, P.; Liu, C. J.; Bai, T.; Li, W. C.; Dai, L. M.; Liu, W. G. Highly Luminescent Carbon Nanodots by MicrowaveAssisted Pyrolysis. Chem. Commun. 2012, 48, 7955-7957.

(46) Shen, P. F.; Xia, Y. S. Synthesis-Modification Integration: OneStep Fabrication of Boronic Acid Functionalized Carbon Dots for Fluorescent Blood Sugar Sensing. Anal. Chem. 2014, 86, 5323-5329.

(47) Liu, C. J.; Zhang, P.; Zhai, X. Y.; Tian, F.; Li, W. C.; Yang, J. H.; Liu, Y.; Wang, H. B.; Wang, W.; Liu, W. G. Nano-Carrier for Gene Delivery and Bioimaging Based on Carbon Dots with PEI-Passivation Enhanced Fluorescence. Biomaterials 2012, 33, 3604-3613.

(48) Liu, R. L.; Wu, D. Q.; Liu, S. H.; Koynov, K.; Knoll, W.; Li, Q. An Aqueous Route to Multicolor Photoluminescent Carbon Dots Using Silica Spheres as Carriers. Angew. Chem., Int. Ed. 2009, 48, 4598-4601.

(49) Qu, S. N.; Wang, X. Y.; Lu, Q. P.; Liu, X. Y.; Wang, L. J. A Biocompatible Fluorescent Ink Based on Water-Soluble Luminescent Carbon Nanodots. Angew. Chem., Int. Ed. 2012, 51, 12215-12218.

(50) Lim, S. Y.; Shen, W.; Gao, Z. Q. Carbon Quantum Dots and Their Applications. Chem. Soc. Rev. 2015, 44, 362-381.

(51) Sun, Y. P.; Zhou, B.; Lin, Y.; Wang, W.; Fernando, K. S.; Pathak, P.; Meziani, M. J.; Harruff, B. A.; Wang, X.; Wang, H. F.; Luo, P. G.; Yang, H.; Kose, M. E.; Chen, B. L.; Veca, L. M.; Xie, S. Y. QuantumSized Carbon Dots for Bright and Colorful Photoluminescence. J. Am. Chem. Soc. 2006, 128, 7756-7757.

(52) Wang, L.; Zhu, S. J.; Wang, H. Y.; Qu, S. N.; Zhang, Y. L.; Zhang, J. H.; Chen, Q. D.; Xu, H. L.; Han, W.; Yang, B. Common Origin of Green Luminescence in Carbon Nanodots and Graphene Quantum Dots. ACS Nano 2014, 8, 2541-2547.

(53) Gan, Z. X.; Wu, X. L.; Hao, Y. L. The Mechanism of Blue Photoluminescence from Carbon Nanodots. CrystEngComm 2014, 16, 4981-4986.

(54) Chen, W.; Tu, X.; Guo, X. Fluorescent Gold NanoparticlesBased Fluorescence Sensor for $\mathrm{Cu}^{2+}$ Ions. Chem. Commun. 2009, $1736-1738$.

(55) Wang, C. X.; Wang, Y.; Xu, L.; Zhang, D.; Liu, M.; Li, X.; Sun, H.; Lin, Q.; Yang, B. Facile Aqueous-Phase Synthesis of Biocompatible and Fluorescent $\mathrm{Ag}_{2} \mathrm{~S}$ Nanoclusters for Bioimaging: Tunable Photoluminescence from Red to Near Infrared. Small 2012, 8, 3137-3142. 
(56) Wang, C. X.; Xu, L.; Xu, X.; Cheng, H.; Sun, H. C.; Lin, Q.; Zhang, C. Near Infrared Ag/Au Alloy Nanoclusters: Tunable Photoluminescence and Cellular Imaging. J. Colloid Interface Sci. 2014, 416, 274-279.

(57) Wang, C. X.; Xu, L.; Wang, Y.; Zhang, D.; Shi, X. D.; Dong, F.; Yu, K.; Lin, Q.; Yang, B. Fluorescent Silver Nanoclusters as Effective Probes for Highly Selective Detection of Mercury(II) at Parts-PerBillion Levels. Chem. - Asian J. 2012, 7, 1652-1656.

(58) Wang, C. X.; Wang, Y.; Xu, L.; Shi, X. D.; Li, X. W.; Xu, X. W.; Sun, H. C.; Yang, B.; Lin, Q. A Galvanic Replacement Route to Prepare Strongly Fluorescent and Highly Stable Gold Nanodots for Cellular Imaging. Small 2013, 9, 413-420.

(59) Chen, Y.; Wang, Y.; Wang, C. X.; Li, W. Y.; Zhou, H. P.; Jiao, H. P.; Lin, Q.; Yu, C. Papain-Directed Synthesis of Luminescent Gold Nanoclusters and the Sensitive Detection of $\mathrm{Cu}^{2+}$. J. Colloid Interface Sci. 2013, 396, 63-68.

(60) Bomm, J.; Günter, C.; Stumpe, J. Synthesis and Optical Characterization of Thermosensitive, Luminescent Gold Nanodots. J. Phys. Chem. C 2012, 116, 81-85.

(61) Maestro, L. M.; Rodríguez, E. M.; Rodríguez, F. S.; Iglesias-de la Cruz, M. C.; Juarranz, A.; Naccache, R.; Vetrone, F.; Jaque, D.; Capobianco, J. A.; Solé, J. G. CdSe Quantum Dots for Two-Photon Fluorescence Thermal Imaging. Nano Lett. 2010, 10, 5109-5115.

(62) Brites, C. D. S.; Lima, P. P.; Silva, N. J. O.; Millán, A.; Amaral, V. S.; Palacio, F.; Carlos, L. D. Thermometry at the Nanoscale. Nanoscale 2012, 4, 4799-4829.

(63) Gan, Z. X.; Wu, X. L.; Zhang, J. L.; Zhu, X. B.; Chu, P. K. In Situ Thermal Imaging and Absolute Temperature Monitoring by Luminescent Diphenylalanine Nanotubes. Biomacromolecules 2013, 14, 2112-2116.

(64) Jethi, L.; Krause, M. M.; Kambhampati, P. Toward Ratiometric Nanothermometry via Intrinsic Dual Emission from Semiconductor Nanocrystals. J. Phys. Chem. Lett. 2015, 6, 718-721.

(65) Liu, J. B.; Yu, M. X.; Ning, X. H.; Zhou, C.; Yang, S. Y.; Zheng, J. PEGylation and Zwitterionization: Pros and Cons in the Renal Clearance and Tumor Targeting of Near-IR-Emitting Gold Nanoparticles. Angew. Chem., Int. Ed. 2013, 52, 12572-12576.

(66) Tao, Y.; Ju, E. G.; Li, Z. H.; Ren, J. S.; Qu, X. G. Engineered CpG-Antigen Conjugates Protected Gold Nanoclusters as Smart SelfVaccines for Enhanced Immune Response and Cell Imaging. Adv. Funct. Mater. 2014, 24, 1004-1010. 